TRANSACTIONS OF THE

AMERICAN MATHEMATICAL SOCIETY

Volume 351, Number 2, February 1999, Pages 813-823

S 0002-9947(99)02043-7

\title{
PROJECTIVE STRUCTURES WITH DISCRETE HOLONOMY REPRESENTATIONS
}

\author{
HIROSHIGE SHIGA AND HARUMI TANIGAWA
}

\begin{abstract}
Let $K(X)$ denote the set of projective structures on a compact Riemann surface $X$ whose holonomy representations are discrete. We will show that each component of the interior of $K(X)$ is holomorphically equivalent to a complex submanifold of the product of Teichmüller spaces and the holonomy representation of every projective structure in the interior of $K(X)$ is a quasifuchsian group.
\end{abstract}

\section{InTRODUCTION}

Let $\Sigma_{g}$ be a compact oriented differentiable surface of genus $g \geq 2$. A projective structure (or $\mathbf{C P}^{1}$-structure) on $\Sigma_{g}$ is a maximal system of charts with transition maps in the projective automorphism group of $\mathbf{C P}^{1}$, namely, $\mathrm{PSL}_{2}(\mathbf{C})$. Let $P_{g}$ denote the space of all projective structures on $\Sigma_{g}$ with markings. Since elements of $\mathrm{PSL}_{2}(\mathbf{C})$ are holomorphic, a projective structure determines its underlying complex structure. Hence there is a natural map $\pi: P_{g} \rightarrow T_{g}$, where $T_{g}$ denotes the Teichmüller space. For each projective structure, we can take its developing map on the universal cover and have a holomorphic quadratic differential (or a projective connection) on the Riemann surface (the underlying complex structure under the projective structure) by taking the Schwarzian derivative. It is well-known (cf. section 2 for detail) that this procedure gives a natural identification between $\pi$ : $P_{g} \rightarrow T_{g}$ and the bundle of holomorphic quadratic differentials over the Teichmüller space $\pi: Q \rightarrow T_{g}$.

In this paper, we will investigate the set of projective structures on $\Sigma_{g}$ with discrete holonomy representations with respect to the parametrization by $Q$. Let $K \subset Q$ be the set of all projective structures with discrete holonomy representations. It is well-known that there is an open set $Q F$ of $Q$, which is a neighborhood of the 0 -section $\{0\} \times T_{g}$ of $Q \rightarrow T_{g}$, consisting of all projective structures with univalent developing maps and quasifuchsian holonomy representations. For any Riemann surface $X \in T_{g}$, the fiber of $\pi \mid Q F: Q F \rightarrow T_{g}$ over $X$, which we denote by $Q F(X)$, is a domain which is holomorphically equivalent to the Teichmüller space and is called a Bers slice. One of our main results shows that a similar phenomenon occurs for each component of the interior point set int $K$.

Let $Q(X)$ denote the fiber over $X$ of $Q \rightarrow T_{g}$, namely, $Q(X)$ is the space of projective structures on the complex structure $X$. Let $K(X)$ denote the fiber of

Received by the editors August 18, 1995 and, in revised form, November 28, 1996 and February $14,1997$.

1991 Mathematics Subject Classification. Primary 32G15; Secondary 30F10.

Research at MSRI is supported by NSF grant \#DMS-9022140.

(C)1999 American Mathematical Society 
$\pi \mid K: K \rightarrow X$. We will denote by $\operatorname{int}_{Q(X)} K(X)$ the interior of $K(X)$ considered in $Q(X)$. We employ this notation to avoid mixing up $\operatorname{int}_{Q(X)} K(X)$ with the fiber of int $K$ (interior is considered in $Q$ ) over $X$, although it will turn out these two are the same by our result. As we recalled above, $\operatorname{int}_{Q(X)} K(X)$ has a component containing the Bers slice $Q F(X)$. (In fact, the Bers slice $Q F(X)$ coincides with the component of $\operatorname{int}_{Q(X)} K(X)$ containing 0 ([Sh]).) On the other hand, it was shown by Maskit $[\mathrm{M}]$ that there are complex structures $X$ on which there exist components of $\operatorname{int}_{Q(X)} K(X)$ other than the Bers slice (see also Hejhal $[\mathrm{H}]$ and Goldman [G2]). Indeed, he showed that given a Fuchsian group isomorphic to $\pi_{1} \Sigma_{g}$ there are projective structures outside the Bers slice on some complex structures whose holonomy representations are conjugate to the given Fuchsian group in $\mathrm{PSL}_{2}(\mathbf{C})$. However, the Fuchsian holonomy representation hardly gives information about the complex structures under the projective structures when the projective structure is not in the Bers slice, because the developing map is not a covering map ([Kr1]). Here arise natural questions:

1. For every complex structure $X \in T_{g}$ are there any components of int $Q(X) K(X)$ other than the Bers slice?

2. What kind of discrete groups appear as holonomy representations in such components?

3. What kind of analytic properties do such components have?

As for the first question, an affirmative answer is given in [T1]. The aim of this paper is to work on the other two questions. We will show that each component of int ${ }_{Q(X)} K(X)$ consists of quasifuchsian groups and is holomorphically equivalent to a properly embedded holomorphic submanifold of the product $T_{g} \times T_{g}$.

The authors would like to thank Curt McMullen for stimulating conversations and comments. Considerable part of this work was done at Mathematical Sciences Research Institute. The authors are very grateful for their hospitality.

Remark. All results of this paper hold for bounded projective structures on Riemann surfaces of finite type (i.e. projective structures determined by bounded holomorphic quadratic differentials) by similar arguments.

\section{NOTATION AND BASIC FACTS}

In this section, we recall some known facts we will use to prove our results.

Throughout this paper, $\Gamma$ denotes a Fuchsian group acting on the upper half plane $\mathbf{H}$ and the lower half plane $L$ such that the quotient $X=L / \Gamma$ is a compact Riemann surface of genus $g \geq 2$.

2.1 Quasiconformal deformations and the Teichmüller space. For a finitely generated Kleinian group $G$, we denote by $\Omega(G)$ its region of discontinuity and by $\Lambda(G)$ its limit set.

For a $G$-invariant open set $U \subset \Omega(G)$, a measurable $(-1,1)$-form $\mu$ on the Riemann sphere with the following properties is called a Beltrami differential for $G$ supported on $U$ :

$$
\mu \mid(\hat{\mathbf{C}}-U)=0, \quad\|\mu\|_{\infty}<1,
$$

and

$$
\mu \circ g \times \overline{g^{\prime}} / g^{\prime}=\mu \quad \text { a.e. }
$$


for all $g \in G$. We denote the set of all Beltrami differentials on $U$ by $B_{1}(G, U)$. When $U=\Omega(G)$, we abbreviate $B_{1}(G, U)$ by $B_{1}(G)$. The space $B_{1}(G)$ is the unit ball of the complex Banach space of all measurable $(-1,1)$-form for $G$ with supremum norm.

By Ahlfors-Bers' theory, for each Beltrami differential $\mu$ on $\hat{\mathbf{C}}$ with $\|\mu\|_{\infty}$, there exists a quasiconformal mapping $f^{\mu}$ with Beltrami differential $\mu$, that is,

$$
\left(f^{\mu}\right)_{\bar{z}}=\mu \times\left(f^{\mu}\right)_{z}
$$

Such a mapping is unique up to post-composition of elements of $\mathrm{PSL}_{2}(\mathbf{C})$. In each argument below, we will employ some convenient normalization.

It is easy to see that if $\mu$ is in $B_{1}(G)$, then $f^{\mu} \circ g \circ\left(f^{\mu}\right)^{-1}$ is a Möbius transformation for every $g \in G$ and $f^{\mu} G\left(f^{\mu}\right)^{-1}$ is a subgroup of $\mathrm{PSL}_{2}(\mathbf{C})$ with region of discontinuity $f^{\mu}(\Omega(G))$.

Two Beltrami differentials $\mu_{1}$ and $\mu_{2}$ for $G$ are said to be equivalent if $f^{\mu_{1}}$ and $f^{\mu_{2}}$ determine equivalent homomorphisms of $G$; namely, there exists a Möbius transformation $A \in \mathrm{PSL}_{2}(\mathbf{C})$ such that

$$
f^{\mu_{1}} \circ g \circ\left(f^{\mu_{1}}\right)^{-1}=A \circ f^{\mu_{2}} \circ g \circ\left(f^{\mu_{2}}\right)^{-1} \circ A^{-1}
$$

for every $g \in G$. A Beltrami differential is called trivial if it is equivalent to 0 .

Definition 2.1. The set of all equivalence classes of Beltrami differentials for $G$ is called the quasiconformal deformation space of $G$ and is denoted by $Q C(G)$.

For a Fuchsian group $\Gamma$ acting on the lower half plane $L$ the Teichmüller space $T(\Gamma)$ of $\Gamma$ is the quotient space of $B_{1}(\Gamma, L)$ by the equivalence relation as above.

The space $Q C(G)$ has a natural complex structure such that the canonical projection $B_{1}(G) \rightarrow Q C(G)$ is holomorphic. Also, the Teichmüller space $T(\Gamma)$ has a natural complex structure such that the quotient map $B_{1}(\Gamma, L) \rightarrow T(\Gamma)$ is holomorphic. The Teichmüller space $T(\Gamma)$ is also regarded as a deformation space of the Riemann surface $X=L / \Gamma$, which we denote by $T(X)$. It is well-known that the complex structure of the Teichmüller space of Riemann surfaces of genus $g$ is independent of the choice of the base point $X$. When we need not mention the base point $X$, we denote the Teichmüller space of Riemann surfaces of genus $g$ by $T_{g}$.

Here, we recall another definition of $T(\Gamma)$ as the space of Fuchsian groups. For $\mu \in B_{1}(\Gamma, L)$, let $f_{\mu}$ denote the quasiconformal homeomorphism of $L$ onto itself with Beltrami differential $\mu \mid L$ fixing 0,1 and $\infty$. The mapping $f_{\mu}$ is the restriction of a quasiconformal mapping $f^{\mu^{\prime}}$ where $\mu^{\prime}$ is a Beltrami differential on $\hat{\mathbf{C}}$ defined by extending $\mu \mid L$ to $\hat{\mathbf{C}}$ symmetrically beyond $\mathbf{R}$ :

$$
\mu^{\prime}(z)= \begin{cases}\mu(z), & \text { if } z \in L \\ \mu(\bar{z}), & \text { if } z \in H\end{cases}
$$

Then the group $f_{\mu} \Gamma\left(f_{\mu}\right)^{-1}$ is a Fuchsian group acting on $L$. It is easy to see that two Beltrami differentials $\mu_{1}$ and $\mu_{2}$ in $B_{1}(\Gamma, L)$ determine the same homomorphism $\Gamma \rightarrow \mathrm{PSL}_{2}(\mathbf{R})$ if and only if $\mu_{1}^{\prime}$ and $\mu_{2}^{\prime}$ are equivalent in $B_{1}(\Gamma)$, where $\mu_{i}^{\prime}(i=1,2)$ is the symmetric extension of $\mu_{i}$ as above. It is also easy to see that $\mu_{1}^{\prime}$ and $\mu_{2}^{\prime}$ are equivalent in $B_{1}(\Gamma)$ if and only if $\mu_{1}$ and $\mu_{2}$ are equivalent in $B_{1}(\Gamma)$.

Definition 2.2 (Teichmüller space as the space of Fuchsian groups). The Teichmüller space $T(\Gamma)$ is the space of equivalence classes of the symmetric Beltrami 
differentials, namely, equivalence classes of Beltrami differentials $\mu^{\prime} \in B_{1}(\Gamma)$ such that $\mu^{\prime}(z)=\overline{\mu^{\prime}(\bar{z})}$ almost everywhere.

By the above remark, this definition of $T(\Gamma)$ is the same as that in Definition 2.1 , if we regard $T(\Gamma)$ as a topological space. Actually, these two definition give the same real analytic structures. However, when we regard $T(\Gamma)$ as the space of Fuchsian groups, it is not considered as a complex manifold, since the space of symmetric Beltrami differentials is not a complex Banach manifold.

See Lehto [L], for example, for more detail on fundamental facts on Teichmüller spaces.

2.2 Projective structures and quadratic differentials. Assume that we are given a projective structure on $\Sigma_{g}$. Since the transition mappings are holomorphic, as they are restrictions of elements of $\mathrm{PSL}_{2}(\mathbf{C})$, we have a complex structure under the projective structure. Let $X$ denote the Riemann surface and let $\Gamma$ be a Fuchsian group acting on $L$ such that $X=L / \Gamma$. Take a coordinate function of the projective structure. We can take its analytic continuation along any curve on $X$ and have a multivalued holomorphic mapping into the Riemann sphere. This multivalued mapping is lifted to a locally univalent meromorphic function $W: L \rightarrow \mathbf{C P}^{1}$ on the universal covering space $L$. This mapping is called a developing map of the projective structure. Note that the developing map is determined by the projective structure uniquely up to post composition of elements with $\mathrm{PSL}_{2}(\mathbf{C})$.

When we take the analytic continuation of a local coordinate function along a closed curve and come back to the starting point, the values differ from each other by elements of $\mathrm{PSL}_{2}(\mathbf{C})$, since the transition mappings are in $\mathrm{PSL}_{2}(\mathbf{C})$. Therefore, we have a homomorphism of $\pi_{1} X$ to $\mathrm{PSL}_{2}(\mathbf{C})$. If we look at this on the universal covering space $L$, we have a homomorphism $\chi: \Gamma \rightarrow \mathrm{PSL}_{2}(\mathbf{C})$ such that

$$
W \circ \gamma(z)=\chi(\gamma) \circ W(z), \quad z \in L,
$$

for all $\gamma \in \Gamma$. This homomorphism is called the holonomy representation.

Thus the projective structure determines the pair $(W, \chi)$ uniquely up to the action of $\mathrm{PSL}_{2}(\mathbf{C})$. Conversely, it is easy to see that given a pair $(W, \chi)$ of locally univalent meromorphic function $W: L \rightarrow \mathbf{C P}^{1}$ and a homomorphism $\chi: \Gamma \rightarrow \mathrm{PSL}_{2}(\mathbf{C})$, where $\Gamma$ is a Fuchsian group, satisfying (2.1) we have a projective structure on the Riemann surface $L / \Gamma$. Therefore, the space of all projective structures on $\Sigma_{g}$ is identified with the set of pairs $(W, \chi)$ satisfying $(2.1)$ modulo the action of $\mathrm{PSL}_{2}(\mathbf{C})$.

Now we recall the parametrization of the space of projective structures by holomorphic quadratic differentials. Given a projective structure on a Riemann surface $X=L / \Gamma$, take the Schwarzian derivative of the developing map $W$ and denote it by $\varphi$. (Here, the Schwarzian derivative of a locally univalent meromorphic function $f$ is defined by $\left(f^{\prime \prime} / f^{\prime}\right)^{\prime}-1 / 2\left(f^{\prime \prime} / f^{\prime}\right)^{2}$.) Then $\varphi$ is a holomorphic function on $L$ satisfying

$$
\varphi=\varphi \circ \gamma \times \gamma^{\prime 2}
$$

for all $\gamma \in \Gamma$. Such a holomorphic function satisfying (2.2) is called a holomorphic quadratic differential for $\Gamma$. A holomorphic quadratic differential for $\Gamma$ is the lift of a holomorphic quadratic differential on the Riemann surface $X=L / \Gamma$. We have seen that given a projective structure on $X$ we have a holomorphic quadratic differential for $\Gamma$. Conversely, given a holomorphic quadratic differential $\varphi$ for $\Gamma$, 
it is well-known (see $[\mathrm{H}]$ or $[\mathrm{Kr} 2]$ ) that there is a locally univalent meromorphic function $W_{\varphi}$ whose Schwarzian derivative is $\varphi$. It is easy to see from (2.1) that there is a homomorphism $\chi_{\varphi}: \Gamma \rightarrow \mathrm{PSL}_{2}(\mathbf{C})$ satisfying (2.1). Thus the space of all projective structures on the surface $\Sigma_{g}$ is identified with the fiber space $Q \rightarrow T_{g}$ of holomorphic quadratic differentials on compact Riemann surfaces of genus $g$.

Notation 2.3. In this paper, we will denote by $W_{\varphi}$ the developing map and by $\chi_{\varphi}$ the holonomy representation of the projective structure determined by $\varphi$. Since they are determined up to elements of $\mathrm{PSL}_{2}(\mathbf{C})$, we have to fix normalization in each argument in the following section. The choice is not essential. We shall employ convenient ones each time.

Notation 2.4. Let Rep denote the space of $\mathrm{PSL}_{2}(\mathbf{C})$ representations of the fundamental group $\pi_{1} X: R e p=\operatorname{Hom}\left(\pi_{1} X, \mathrm{PSL}_{2}(\mathbf{C})\right) / \mathrm{PSL}_{2}(\mathbf{C})$. Here, two $\mathrm{PSL}_{2}(\mathbf{C})$ representations are equivalent if they differ by conjugation in $\mathrm{PSL}_{2}(\mathbf{C})$. We define a mapping $h: Q \rightarrow R e p$ by sending each $\varphi \in Q$ to the holonomy representation of the projective structure determined by $\varphi$, and call it the holonomy map.

We will use the following property of $h$ :

Theorem 2.5 (Hejhal [H, Theorem 1]). The mapping $h: Q \rightarrow$ Rep is not a covering map. However, it is a local $C^{1}$-diffeomorphism.

Remark. When we say $h: Q \rightarrow R e p$ is a local diffeomorphism, we have to be careful as the space Rep is not even a Hausdorff space. However, the image of $h: Q \rightarrow R e p$ is contained in a smooth manifold. See $[\mathrm{H}]$.

Note that when we regard $Q$ as the space of quadratic differentials for Fuchsian groups on $L$, we regard $T(\Gamma)$ as the space of Fuchsian groups, hence not a complex manifold. In particular, the vector bundle $Q \rightarrow T(\Gamma)$ is not holomorphic. However, each fiber $Q\left(\Gamma^{\prime}\right)$ over $\Gamma^{\prime} \in T(\Gamma)$ has a natural complex structure as a finite dimensional complex linear space.

Lemma 2.6. The holonomy map $h: Q(\Gamma) \rightarrow$ Rep is holomorphic for each $\Gamma$.

Note that the fiber $Q(\Gamma)$ is identified with the space of projective structures whose underlying complex structure is $X=L / \Gamma$.

In the next section, we will discuss the space of projective structures with discrete holonomy representations. We denote by $K$ the subset of $Q$ consisting of all elements $\varphi$ which determine projective structures with discrete holonomy representations and denote by $K(\Gamma)$ the fiber over a point $\Gamma$. We denote by $\operatorname{int}_{Q(\Gamma)} K(\Gamma)$ the interior of $K(\Gamma)$ considered in $Q(\Gamma)$.

The set of all equivalence classes of $B_{1}(\Gamma, H)$ is called the Bers slice. From the definition the Bers slice is isomorphic to the Teichmüller space. For each element $\mu \in B_{1}(\Gamma, H), f^{\mu} \mid L$ is a univalent meromorphic mapping and $f^{\mu} \Gamma\left(f^{\mu}\right)^{-1}$ is a quasifuchsian group. Therefore, it determines a projective structure on $L / \Gamma$. Thus the Bers slice is embedded in $Q(\Gamma)$. We will also call this image Bers slice.

As we noted in the introduction, for each $\Gamma$, $\operatorname{int}_{Q(\Gamma)} K(\Gamma)$ has a component which contains the Bers slice.

Proposition 2.7 ([Sh]). The component of $\operatorname{int}_{Q(\Gamma)} K(\Gamma)$ containing 0 coincides with the Bers slice. For any component $\kappa(\Gamma)$ of $\operatorname{int}_{Q(\Gamma)} K(\Gamma)$ which is not the Bers slice, the developing map $W_{\varphi}$ is surjective onto the Riemann sphere for every $\varphi \in \kappa(\Gamma)$. For any two elements $\varphi_{1}$ and $\varphi_{2}$ of $\kappa(\Gamma), \chi_{\varphi_{1}}(\Gamma)$ and $\chi_{\varphi_{2}}(\Gamma)$ are quasi-conformally equivalent to each other. 
2.3 Holomorphic motions of Kleinian groups. Here we state the theorem of holomorphic motions in a convenient form for later use. (See [EKK] for details and references for the theory of holomorphic motions.)

Definition 2.8. Let $G$ be a finitely generated discrete group of $\mathrm{PSL}_{2}(\mathbf{C})$. A family $\left\{\theta_{z}\right\}_{z \in \Delta}$ of isomorphisms of $G$ into $\mathrm{PSL}_{2}(\mathbf{C})$, parametrized by the unit disc $\Delta=$ $\{z \in \mathbf{C} ;|z|<1\}$, is called a holomorphic motion of $G$ or a holomorphic family of isomorphisms of $G$ if it satisfies the following conditions:

1. $\theta_{0}$ is the identity: $\theta_{0}(g)=g$ for all $g \in G$.

2. For every $z \in \Delta, \theta_{z}(G)$ is discrete.

3. For every $g \in G, z \mapsto \theta_{z}(g)$ is a holomorphic mapping of $\Delta$ to $\mathrm{PSL}_{2}(\mathbf{C})$.

Theorem 2.9 (Bers [B], Earle-Kra-Krushkal [EKK], and Slodkowski [Sl]). Let $\left\{\theta_{z}\right\}_{z \in \Delta}$ be a holomorphic family of isomorphisms of a discrete group $G$. Then there is a holomorphic mapping $z \mapsto \mu_{z}$ from $\Delta$ to $B_{1}(G)$ such that $\theta_{z}(\gamma)=$ $f^{\mu_{z}} \circ \gamma \circ\left(f^{\mu_{z}}\right)^{-1}$ for all $\gamma \in G$ where $f^{\mu_{z}}$ is a quasiconformal mapping with Beltrami differential $\mu_{z}$.

Remark. If we have a holomorphic family of discrete groups, it is actually a holomorphic family of discontinuous groups, unless the family is trivial. In fact, by the above theorem the holomorphic family of isomorphisms is given by a family of quasiconformal deformations. If the discrete group is not discontinuous, there is no non-trivial quasiconformal deformations by Sullivan's rigidity theorem ([Su]). Therefore, $\operatorname{int}_{Q(\Gamma)} K(\Gamma)$ coincides with the interior of set of projective structures with discontinuous holonomy representations.

\section{Projective structures with discrete holonomy Representations}

To prove the main result, we will construct a local inverse to the holonomy map $h: Q \rightarrow R e p$ on each component of int $K$, which is holomorphic in the direction of fibers of $Q \rightarrow T_{g}$. For the construction, we first note the following fact.

Lemma 3.1. Each component $\kappa(\Gamma)$ of $\operatorname{int}_{Q(\Gamma)} K(\Gamma)$ either consists of totally degenerate groups or consists of quasifuchsian groups which are isomorphic to $\Gamma$.

Proof. First, note that for every $\varphi \in \kappa(\Gamma) \chi_{\varphi}$ is an isomorphism which preserves parabolicity (Kra [Kr2] and [Kr3]).

By the result of Maskit [M, Theorem 2 and Theorem 3], $\chi_{\varphi}(\Gamma)$ is either a quasifuchsian group or a totally degenerate group. By Proposition 2.7, either $\chi_{\varphi}(\Gamma)$ is a quasifuchsian group for every $\varphi \in \kappa(\Gamma)$ or $\chi_{\varphi}(\Gamma)$ is a totally degenerate group for every $\varphi \in \kappa(\Gamma)$.

We now define a mapping which plays a key role in analyzing the structure of $\operatorname{int} K$ or $\operatorname{int}_{Q(\Gamma)} K(\Gamma)$.

Fix an element $\varphi \in \kappa(\Gamma)$ and denote the holonomy group $\chi_{\varphi}(\Gamma)$ by $G_{\varphi}$, for simplicity. For each Beltrami differential $\mu \in B_{1}\left(G_{\varphi}\right)$ for $G_{\varphi}$, we pull back $\mu$ via the developing map $W_{\varphi}$ to a Beltrami differential $\hat{\mu}$; namely, we set

$$
\hat{\mu}=\mu \circ W_{\varphi} \times \overline{W_{\varphi}^{\prime}} / W_{\varphi}^{\prime} .
$$

The differential $\hat{\mu}$ is the Beltrami differential of the local homeomorphism $f^{\mu}$ 。 $W_{\varphi}$, where $f^{\mu}$ is a quasiconformal mapping with Beltrami differential $\mu$. It is easy to see that $\hat{\mu}$ is an element of $B_{1}(\Gamma, L)$, and the assignment $\hat{\Phi}: \mu \rightarrow \hat{\mu}$ is a holomorphic mapping from $B_{1}\left(G_{\varphi}\right)$ to $B_{1}(\Gamma, L)$. 
Proposition 3.2. In the above situation, the mapping $\hat{\Phi}: \mu \rightarrow \hat{\mu}$ descends to a holomorphic mapping $\Phi: Q C\left(G_{\varphi}\right) \rightarrow T(\Gamma)$. (Here, we regard $T(\Gamma)$ as a complex manifold.)

Proof. We have to show that two equivalent elements $\mu_{1}$ and $\mu_{2}$ in $B_{1}\left(G_{\varphi}\right)$ are mapped by $\hat{\Phi}$ to equivalent elements $\hat{\mu}_{1}$ and $\hat{\mu}_{2}$ in $B_{1}(\Gamma, L)$.

We first show that every trivial differential $\tau$ in $B_{1}\left(G_{\varphi}\right)$ is pulled back to a trivial differential $\hat{\tau}$ in $B_{1}(\Gamma, L)$. Choose three points in $\Lambda\left(G_{\varphi}\right)$. For each $\mu \in B_{1}\left(G_{\varphi}\right)$, let $f^{\mu}$ denote the quasiconformal mapping with Beltrami differential $\mu$ which fixes these three points. If $\tau$ is trivial, then $f^{\tau}$ fixes every point on $\Lambda\left(G_{\varphi}\right)$ and maps each component of $\Omega\left(G_{\varphi}\right)$ onto itself. Below we describe how the locally univalent mapping $W_{\varphi}$ for $\varphi \in \operatorname{int}_{Q(\Gamma)} K(\Gamma)$ behaves in $L$. The preimages $\left(W_{\varphi}\right)^{-1}\left(\Omega\left(G_{\varphi}\right)\right)$ and $\left(W_{\varphi}\right)^{-1}\left(\Lambda\left(G_{\varphi}\right)\right)$ are $\Gamma$ invariant and mutually disjoint. By Lemma 3.1, the holonomy representation $G_{\varphi}$ is either a quasifuchsian group or a totally degenerate group. In either case, each component of $\Omega\left(G_{\varphi}\right)$ is simply connected. Hence each germ of the local inverse of $W_{\varphi}$ has an analytic continuation in each component of $\Omega\left(G_{\varphi}\right)$. Therefore each component of the preimage $\left(W_{\varphi}\right)^{-1}\left(\Omega\left(G_{\varphi}\right)\right)$ is a simply connected domain which is mapped onto a component of $\Omega\left(G_{\varphi}\right)$ by $W_{\varphi}$ injectively. Decompose $W_{\varphi}^{-1}\left(\Omega\left(G_{\varphi}\right)\right)$ to the disjoint union of connected components and denote it by $\bigcup_{n=1}^{\infty} D_{\varphi, n}$. As we have seen above for each $n$ there is a branch $W_{\varphi, n}^{-1}$ of $W_{\varphi}^{-1}$ on $W_{\varphi}\left(D_{\varphi, n}\right)$ such that $W_{\varphi, n}^{-1} \circ W_{\varphi}=i d$. on $D_{\varphi, n}$.

We define a mapping $f: L \rightarrow L$ by

$$
f(z)= \begin{cases}z & \text { on } W_{\varphi}^{-1}\left(\Lambda\left(G_{\varphi}\right)\right), \\ W_{\varphi, n}^{-1} \circ f^{\tau} \circ W_{\varphi}(z) & \text { on } D_{\varphi, n} \quad(n=1,2, \ldots) .\end{cases}
$$

Now we claim that $f$ is a quasiconformal homeomorphism of $L$. (Intuitively, $f$ is nothing but $f^{\tau}$ with the local charts given by $W_{\varphi}$ rather than the standard coordinates of the Riemann sphere.) We have to care about the behavior of $f$ near $W_{\varphi}^{-1}\left(\Lambda\left(G_{\varphi}\right)\right)$.

Fix a point $z_{0} \in W_{\varphi}^{-1}\left(\Lambda\left(G_{\varphi}\right)\right)$ arbitrarily and take a small disc $\delta$ around $z_{0}$. The image $W_{\varphi}(\delta)$ is a neighborhood of $W_{\varphi}\left(z_{0}\right) \in \Lambda\left(G_{\varphi}\right)$. Take a disc $\delta^{\prime} \ni z_{0}$ small enough so that $f^{\tau}\left(W_{\varphi}\left(\delta^{\prime}\right)\right) \subset W_{\varphi}(\delta)$. Recall that $f^{\tau} \mid W_{\varphi}(\delta) \cap \Lambda\left(G_{\varphi}\right)=i d$. We can take the branch $W_{\varphi, \delta^{\prime}}^{-1}$ of $W_{\varphi}^{-1}$ on $W_{\varphi}\left(\delta^{\prime}\right)$ such that $W_{\varphi, \delta^{\prime}}^{-1} \circ W_{\varphi}$ is the identity on $\delta^{\prime}$. With this branch of $W_{\varphi}^{-1}$, we can define a quasiconformal mapping by $W_{\varphi, \delta^{\prime}}^{-1} \circ f^{\tau} \circ W_{\varphi}$ on $\delta^{\prime}$.

Obviously, $W_{\varphi, \delta^{\prime}}^{-1} \circ f^{\tau} \circ W_{\varphi}$ coincides with $f$ on $W_{\varphi}^{-1}\left(\Lambda\left(G_{\varphi}\right)\right)$. We claim that this mapping coincides with the mapping $W_{\varphi, n}^{-1} \circ f^{\tau} \circ W_{\varphi}$ also on each component of $D_{\varphi, n} \cap \delta^{\prime}$. Then it will follow that $f=W_{\varphi, \delta^{\prime}}^{-1} \circ f^{\tau} \circ W_{\varphi}$ is quasiconformal near $z_{0}$. (Note that this claim is not quite obvious, because $W_{\varphi}\left(\delta^{\prime}\right) \cap \Omega\left(G_{\varphi}\right)$ may have infinitely many components and $f^{\tau}$ might exchange these components.) In order to show the above claim, it suffices to show that $f^{\tau}$ maps each component of $W_{\varphi}\left(\delta^{\prime}\right) \cap \Omega\left(G_{\varphi}\right)$ into the component of $W_{\varphi}(\delta) \cap \Omega\left(G_{\varphi}\right)$ containing it. To verify this, we take an isotopy $\left\{f_{t}\right\}_{0 \leq t \leq 1}$ between $f^{\tau}$ and the identity map via quasiconformal mappings fixing every point of $\Lambda\left(G_{\varphi}\right)$; here, the existence of such an isotopy is due to Earle-McMullen [EM]. Replacing $\delta^{\prime}$ into a smaller disc, if necessary, we may assume that $f_{t}\left(W_{\varphi}\left(\delta^{\prime}\right)\right) \subset W_{\varphi}(\delta)$ for all $t \in[0,1]$. Then for each component $u$ of $W_{\varphi}\left(\delta^{\prime}\right) \cap \Omega\left(G_{\varphi}\right)$ and $t, f_{t}(u)$ is contained in a component of $W_{\varphi}(\delta) \cap \Omega\left(G_{\varphi}\right)$ and the component depends continuously on $t$. Hence it is independent of $t$. 
Thus we have seen that $f$ is a quasiconformal homeomorphism of $L$ with Beltrami differential $\hat{\tau}$. Since $f$ is the identity map on $W_{\varphi}^{-1}\left(\Lambda\left(G_{\varphi}\right)\right)$ and since this set is $\Gamma$ invariant, the extension of $f$ to the real axis is the identity. Hence $\hat{\tau}$ is a trivial differential.

Now we proceed to the general case. Assume that $\mu_{1}$ and $\mu_{2}$ are equivalent in $B_{1}\left(G_{\varphi}\right)$. Then there is a trivial differential $\tau$ in $B_{1}\left(G_{\varphi}\right)$ such that $f^{\mu_{2}}=f^{\mu_{1}} \circ f^{\tau}$. Now, as we have shown above, $\hat{\tau}$ is trivial in $B_{1}(\Gamma, L)$ and $W_{\varphi} \circ f_{\hat{\tau}}=f^{\tau} \circ W_{\varphi}$, where $f_{\hat{\tau}}$ is the quasiconformal homeomorphism of $L$ with Beltrami differential $\hat{\tau}$ fixing 0,1 and $\infty$. Hence we have

$$
\begin{aligned}
f^{\mu_{2}} \circ W_{\varphi} & =f^{\mu_{1}} \circ f^{\tau} \circ W_{\varphi} \\
& =f^{\mu_{1}} \circ W_{\varphi} \circ f_{\hat{\tau}} .
\end{aligned}
$$

Let $f_{\hat{\mu}_{i}}, i=1,2$, denote the quasiconformal homeomorphism of $L$ with Beltrami differential $\hat{\mu}_{i}$ fixing 0,1 and $\infty$. Since the Beltrami differential $\hat{\mu}_{i}$ is the Beltrami differential of $f^{\mu_{i}} \circ W_{\varphi}$ by definition, the above equality implies

$$
f_{\hat{\mu}_{2}}=f_{\hat{\mu}_{1}} \circ f_{\hat{\tau}} \text {. }
$$

Since $\hat{\tau}$ is trivial, $\hat{\mu}_{1}$ and $\hat{\mu}_{2}$ are equivalent.

We define another mapping which is essentially obtained in the above argument.

Fix a point $\varphi \in \operatorname{int}_{Q(\Gamma)} K(\Gamma)$. For each $\mu \in B_{1}\left(G_{\varphi}\right)$, let $f_{\hat{\mu}}$ denote the quasiconformal homeomorphism of $L$ with the Beltrami differential $\hat{\mu}$ fixing 0,1 and $\infty$. Then by the definition of $\hat{\mu}$, the mapping $W^{\mu}$ defined by $W^{\mu}=f^{\mu} \circ W_{\varphi} \circ f_{\hat{\mu}}^{-1}$ is a locally univalent holomorphic mapping on $L$ to the Riemann sphere. Moreover,

$$
W^{\mu} \circ\left(f_{\hat{\mu}} \circ \gamma \circ f_{\hat{\mu}}^{-1}\right)=\left(f^{\mu} \circ \chi_{\varphi}(\gamma) \circ\left(f^{\mu}\right)^{-1}\right) \circ W^{\mu}
$$

for all $\gamma \in \Gamma$. Thus $W^{\mu}$ is a developing map of a projective structure on $L / \Gamma_{\hat{\mu}}$ where $\Gamma_{\hat{\mu}}=f_{\hat{\mu}} \Gamma f_{\hat{\mu}}^{-1}$.

Taking the Schwarzian derivative of $W^{\mu}$, we have a holomorphic quadratic differential $\tilde{\phi}(\mu) \in Q\left(\Gamma_{\mu}\right)$. Thus we have a mapping $\tilde{\phi}: B_{1}\left(G_{\varphi}\right) \rightarrow Q$. The mapping $\tilde{\phi}: B_{1}\left(G_{\varphi}\right) \rightarrow Q$ descends to a mapping from $Q C\left(G_{\varphi}\right)$ into $Q$. In fact, if $\mu_{1}$ and $\mu_{2}$ are equivalent, $\hat{\mu}_{1}$ and $\hat{\mu}_{2}$ are equivalent by Proposition 3.2. Therefore we have $\Gamma_{\hat{\mu}_{1}}=\Gamma_{\hat{\mu}_{2}}$ and $\tilde{\phi}\left(\hat{\mu}_{1}\right)$ and $\tilde{\phi}\left(\hat{\mu}_{2}\right)$ are in the same fiber $Q\left(\Gamma_{\hat{\mu}_{1}}\right)$. Furthermore, these two quadratic differentials determine projective structures with the same holonomy representation. Hence by the following theorem by Poincaré (cf. Kra [Kr1]), these holomorphic differential are the same.

Theorem (Poincaré). Two different projective structures on the same complex structure of a compact surface have different holonomy representations.

We shall denote the mapping $Q C\left(G_{\varphi}\right) \rightarrow Q$ by $\phi$. The mapping $\phi: Q C\left(G_{\varphi}\right) \rightarrow$ $Q$ is a continuous mapping which satisfies $\phi \circ h=i d$, where $h$ is the holonomy map. By Theorem 2.5, $\phi$ is a $C^{1}$ map. Also, it satisfies $\Phi=\pi \circ \phi$, where $\pi: Q \rightarrow T(\Gamma)$ denotes the projection and $\Phi: Q C\left(G_{\varphi}\right) \rightarrow T(\Gamma)$ is the holomorphic mapping defined in Proposition 3.2.

Proposition 3.3. Let $\varphi$ be an element of $\operatorname{int}_{Q(\Gamma)} K(\Gamma)$ and let $\kappa_{\varphi}(\Gamma)$ denote the component of $\operatorname{int}_{Q(\Gamma)} K(\Gamma)$ containing $\varphi$. Then $\kappa_{\varphi}(\Gamma)$ is a subset of a component of $\phi\left(Q C\left(G_{\varphi}\right)\right) \cap Q(\Gamma)$. 
Proof. We shall show every element $\psi \in \kappa_{\varphi}(\Gamma)$ is contained in $\phi\left(Q C\left(G_{\varphi}\right)\right) \cap Q(\Gamma)$. First, we consider the case that $\psi$ is close to $\varphi$ so that there is a complex analytic disc connecting them; namely, there is a family $\left\{\varphi_{z}\right\}_{z \in \Delta} \subset \kappa_{\varphi}(\Gamma)$ depending holomorphically on $z$ such that $\varphi=\varphi_{0}$ and $\varphi_{\zeta}=\psi$ for some $\zeta \in \Delta$ (here, $\Delta$ denotes the unit disc of the complex plane). Then the set of holonomy representations $\chi_{\varphi_{z}}$ depends holomorphically on $z$. Note that $\chi_{\varphi_{z}}$ is isomorphic by Lemma 3.1. Applying Theorem 2.9 to the family $\chi_{\varphi_{z}} \circ \chi_{\varphi_{0}}^{-1}$, we have a family of Beltrami differentials $\left\{\mu_{z}\right\}$ depending holomorphically on $z$ such that

$$
\chi_{\varphi_{z}}(\gamma)=f^{\mu_{z}} \circ \chi_{\varphi}(\gamma) \circ\left(f^{\mu_{z}}\right)^{-1},
$$

for all $\gamma \in \Gamma$ and $z \in \Delta$. For the Beltrami differential $\hat{\mu}_{z}=\hat{\Phi}\left(\mu_{z}\right)$ and the quadratic differential $\phi\left(\mu_{z}\right) \in Q\left(\Gamma_{\hat{\mu}_{z}}\right)$ we shall show that $\phi\left(\mu_{z}\right)=\varphi_{z}$ for all $z \in \Delta$. The set $\left\{z \in \Delta ; \phi\left(\mu_{z}\right)=\varphi_{z}\right\}$ is clearly a closed set and non-empty. (It contains 0 .) On the other hand, since $\phi\left(\mu_{z}\right)$ and $\varphi_{z}$ have the same holonomy representation and the representation map $h: Q \rightarrow R e p$ is a local homeomorphism (Theorem 2.5), the set $\left\{z \in \Delta ; \phi\left(\mu_{z}\right)=\varphi_{z}\right\}$ is also an open set. Hence $\phi\left(\mu_{z}\right)=\varphi_{z}$ for all $z \in \Delta$.

Thus we have shown that all $\psi \in \kappa_{\varphi}(\Gamma)$ sufficiently close to $\varphi$ are contained in $Q(\Gamma) \cap \phi\left(Q C\left(G_{\varphi}\right)\right)$. For general $\psi \in \kappa_{\varphi}(\Gamma)$, we take a chain of holomorphic discs connecting $\psi$ and $\varphi$ and apply the above argument to each disc.

Now we can prove the main theorem.

Theorem 3.4. Every component of $\operatorname{int}_{Q(\Gamma)} K(\Gamma)$ consists of quasifuchsian groups. Every such component is holomorphically equivalent to a complex analytic submanifold of $T_{g} \times T_{g}$.

Proof. To prove the first claim, it is sufficient to show that there is no component of int $_{Q(\Gamma)} K(\Gamma)$ consisting of totally degenerate groups, by Lemma 3.1.

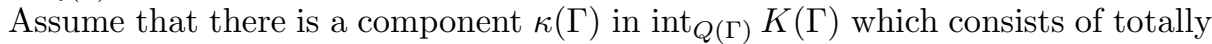
degenerate groups. We will draw a contradiction. Take an element $\varphi \in \kappa(\Gamma)$ and denote the holonomy group by $G_{\varphi}$ for short. We define a mapping $\Phi: Q C\left(G_{\varphi}\right) \rightarrow$ $T(\Gamma)$ as in Proposition 3.2. Then since $\kappa(\Gamma)$ and $Q C\left(G_{\varphi}\right)$ are of the same dimension, $h(\kappa(\Gamma))$ is an open set of $Q C\left(G_{\varphi}\right)$. It follows that the holomorphic mapping $\Phi$ : $Q C\left(G_{\varphi}\right) \rightarrow T(\Gamma)$ takes the value $\Gamma$ on the open set $h(\kappa(\Gamma))$ of $Q C\left(G_{\varphi}\right)$. Therefore it is a constant mapping: $\Phi\left(Q C\left(G_{\varphi}\right)\right) \equiv \Gamma$. It follows that $\phi\left(Q C\left(G_{\varphi}\right)\right) \subset Q(\Gamma)$. As $Q C\left(G_{\varphi}\right)$ and $Q(\Gamma)$ are of the same dimension, $\phi\left(Q C\left(G_{\varphi}\right)\right)$ is an open subset of $Q(\Gamma)$. From this fact and Proposition 3.3, we have $\phi\left(Q C\left(G_{\varphi}\right)\right)=\kappa(\Gamma)$.

It follows that there is a point $\psi \in \kappa(\Gamma)$ such that the holonomy representation $\chi_{\psi}(\Gamma) \in Q C\left(G_{\varphi}\right)$ has Fuchsian equivalent $\Gamma$. Namely, we can choose a point $f^{\mu} G_{\varphi}\left(f^{\mu}\right)^{-1} \in Q C\left(G_{\varphi}\right)$ such that there is a univalent holomorphic mapping $W: L \rightarrow \Omega\left(f^{\mu} G_{\varphi}\left(f^{\mu}\right)^{-1}\right)$ such that $W \circ \gamma=f^{\mu} \chi_{\varphi}(\gamma)\left(f^{\mu}\right)^{-1} \circ W$ for all $\gamma \in \Gamma$ and $\psi=\phi(\mu)$. Now, the projective structure defined by the univalent map $W$ and the projective structure defined by $W_{\psi}$ have the same holonomy representation and have the same underlying complex structure. By Poincaré's theorem, $W$ and $W_{\psi}$ coincide up to Möbius transformation. However, this is impossible because $W(L)=\Omega\left(f^{\mu} G_{\varphi}\left(f^{\mu}\right)^{-1}\right) \varsubsetneqq \hat{\mathbf{C}}$, while $W_{\psi}(L)=\hat{\mathbf{C}}$ by Proposition 2.7. (Note that $\kappa(\Gamma)$ is not the Bers slice.)

We have seen that each component of $\operatorname{int}_{Q(\Gamma)} K(\Gamma)$ consists of projective structures whose holonomy representations are quasifuchsian. From this fact, we shall show that $\kappa_{\varphi}(\Gamma)$ coincides with the component of $\phi\left(Q C\left(G_{\varphi}\right)\right) \cap Q(\Gamma)$ containing 
$\varphi$. Recall the proof of Proposition 3.3. For each point $\varphi \in \operatorname{int}_{Q(\Gamma)} K(\Gamma)$, the component $\kappa_{\varphi}(\Gamma)$ of $\operatorname{int}_{Q(\Gamma)} K(\Gamma)$ containing $\varphi$ is an open subset of a component of $\phi\left(Q C\left(G_{\varphi}\right)\right) \cap Q(\Gamma)$. On the other hand, as $G_{\varphi}$ is a quasifuchsian group, $Q$ and $Q C\left(G_{\varphi}\right)$ have the same dimension. Therefore, $h^{-1}\left(Q C\left(G_{\varphi}\right)\right)$ is an open subset of $Q$; hence each component of $\phi\left(Q C\left(G_{\phi}\right)\right) \cap Q(\Gamma)$ is an open subset of $\operatorname{int}_{Q(\Gamma)} K(\Gamma)$. Therefore, $\kappa_{\varphi}(\Gamma)$ coincides with the component of $\phi\left(Q C\left(G_{\varphi}\right)\right) \cap Q(\Gamma)$ containing $\varphi$.

It follows that $\kappa_{\varphi}(\Gamma)$ is biholomorphically equivalent to the component of $\Phi^{-1}(\Gamma)$ containing $G_{\varphi}$, which is a submanifold of $Q C\left(G_{\varphi}\right)$ by Lemma 2.6. Now the second claim of the theorem follows from the fact that $Q C\left(G_{\varphi}\right)$ is holomorphically equivalent to $T_{g} \times T_{g}$.

What we have done above is to embed $Q C\left(G_{\varphi}\right)$ into $Q$ by the mapping $\phi$ (in fact, we have seen that $\phi$ is a local inverse to the holonomy map) and consider the slice along a fiber of $Q \rightarrow T_{g}$. It is easy to see that for every $\Gamma^{\prime} \in T(\Gamma)$ each component of the slice over $\Gamma^{\prime}$ is a component of $\operatorname{int}_{Q(\Gamma)} K\left(\Gamma^{\prime}\right)$

Corollary 3.5. Each component of $\operatorname{int}_{Q(\Gamma)} K(\Gamma)$ is complete with respect to the Kobayashi hyperbolic metric and Carathéodory distance. Hence each such component is a domain of holomorphy.

Proof. We showed in the above theorem that each component $\kappa(\Gamma)$ of $\operatorname{int}_{Q(\Gamma)} K(\Gamma)$ is holomorphically equivalent to a submanifold $T_{g} \times T_{g}$. It is well-known that $T_{g}$ is complete with respect to the Carathéodory distance and Kobayashi metric. By a standard argument (see Kobayashi $[\mathrm{Ko}]$ ), $T_{g} \times T_{g}$ and its submanifolds are complete with respect to these metrics. It is also known that in general a domain in $\mathbf{C}^{N}$ complete with respect to Carathéodory distance is a domain of holomorphy.

Here we give a brief remark on the existence of components of $\operatorname{int}_{Q(X)} K(X)$ other than the Bers slice, which is discussed in [T1]. In the above discussion, we have shown that any component of $\operatorname{int}_{Q(\Gamma)} K(\Gamma)$ is a component of int $K \cap Q(\Gamma)$. It is shown in [T2] that int $K$ has infinitely many components and each component has non-empty intersection with $Q(\Gamma)$. Therefore we have:

Theorem 3.6. On any complex structure $X \in T_{g}$ there are infinitely many components of $\operatorname{int}_{Q(X)} K(X)$.

\section{REFERENCES}

[B] L. Bers, Holomorphic families of isomorphisms of Möbius groups, J. Math. Kyoto Univ. 26 (1986), 73-76. MR 87j:32067

[EKK] C. Earle, I. Kra and S. Krushkal, Holomorphic Motions and Teichmüller spaces, Trans. Amer. Math. Soc. 343 (1994), 927-948. MR 94h:32035

[EM] C. Earle and C. McMullen, Quasiconformal isotopes, Holomorphic Functions and Moduli II, Springer-Verlag, New York, Berlin, Heidelberg, London, Paris and Tokyo, 1988, pp. 143154. MR 89c:30002

[G1] W. Goldman, The symplectic nature of fundamental groups of surfaces, Advances in Math. 54 (1984), 200-225. MR 86i:32042

[G2] _ Projective structures with Fuchsian holonomy, J. Diff. Geom 25 (1987), 297-326. MR 88i:57006

[H] D. Hejhal, Monodromy groups and linearly polymorphic functions, Acta Math. 135 (1975), 1-55. MR 57:3380

[JM] D. Johnson and J. Millson, Deformation spaces associated to compact hyperbolic manifolds, Discrete Groups and Analysis, Papers in honor of G. D. Mostow on his 60-th birthday, Progress in Mathematics, vol. 67, Birkhäuser, pp. 48-106. MR 88j:22010 
[Ko] S. Kobayashi, Hyperbolic Manifolds and Holomorphic mappings, Marcel Dekker Inc., New York, 1970. MR 43:3503

[Kr1] I. Kra, A generalization of a theorem of Poincaré, Proc. Amer. Math. Soc. 27 (1971), 299-302. MR 46:347

[Kr2] — Deformations of Fuchsian groups, Duke Math. J. 36 (1969), 537-546. MR 42:491

[Kr3] _ Deformations of Fuchsian groups II, Duke Math. J. 38 (1971). MR 44:2951

[L] O. Lehto, Univalent functions and Teichmüller spaces, Springer-Verlag, New York, Berlin, Heidelberg, London, Paris and Tokyo, 1985. MR 88f:30073

[M] B. Maskit, On a class of Kleinian groups, Ann. Acad. Sci. Fenn. Ser. A 442 (1969), 1-8. MR 40:5857

[Sh] H. Shiga, Projective structures on Riemann surfaces and Kleinian groups, J. Math. Kyoto Univ. 27 (1987), 433-438. MR 88k:30056

[Sl] Z. Slodkowski, Holomorphic motions and polynomial hulls, Proc. Amer. Math. Soc. 111 (1991), 347-355. MR 91f:58078

[Su] D. Sullivan, On the ergodic theory at infinity of an arbitrary discrete group of hyperbolic motions, Riemann Surfaces and Related Topics: Proceedings of the 1978 Stony Brook Conference, Annals of Math. Studies 97, Princeton, 1981. MR 83f:58032

[T1] H. Tanigawa, Grafting, harmonic maps and projective structures on surfaces, J. Differential Geometry 47 (1997), 399-419. CMP 98:11

[T2] , Divergence of projective structures and lengths of measured laminations, (preprint).

Department of Mathematics, Tokyo Institute of Technology, Tokyo 152 Japan

E-mail address: shiga@math.titech.ac.jp

Graduate School of Polymathematics, Nagoya University, Nagoya 464-01 Japan

E-mail address: harumi@math.nagoya-u.ac.jp 